IDDF2019-ABS-0160 CATHEPSIN C PROMOTES TUMOR GROWTH AND METASTASIS THROUGH ACTIVATING TNF- $\alpha /$ MAPK (P38) PATHWAY IN HEPATOCELLULAR CARCINOMA

Guo-Pei Zhang*, Xiao Yue, Shao-Qiang Li. The First Affiliated Hospital of Sun Yat-sen University, China

\subsection{6/gutjnl-2019-IDDFabstracts.98}

Background Although cathepsin C (CTSC), a multifunctional molecule, has been reported to maintain various malignant biological properties in several types of cancers, the potential function of CTSC in HCC remains obscure. We aimed to investigate the potential role of cathepsin C (CTSC) in the tumorigenesis of HCC.

Methods One hundred and twenty-two HCC specimens upon tissue microarrays were employed to analyze the correlation between CTSC expression and clinicopathological characteristics through immunohistochemistry staining. qRT-PCR, western blot assay, CCK-8 assay, colony formation, cell migration and invasion assays, xenograft mice model were adopted to validate what had been indicated by the bioinformatic web tools.

Results In our study, by the bioinformatic tools and tissue microarrays, CTSC was found upregulated in HCC compared with normal liver tissues, and its higher expression was correlated with poor prognosis of HCC patients. By gain-of-function and loss-of-function studies, we implicated that CTSC functioned as an oncogene to promote the proliferation, migration and invasion of HCC cells. Mechanistically, we revealed that CTSC was involved in several cancer-related signaling pathways by Gene Set Enrichment Analysis (GSEA), among which TNF- $\alpha / \mathrm{p} 38$ pathway was verified to be activated by CTSC. Furthermore, we found that TNF- $\alpha$ could activate CTSC expression in a concentration-dependent manner. Ralimetinib, an oral MAPK (p38) inhibitor could inhibit CTSC expression. These indicated a potential positive feedback loop between CTSC and TNF- $\alpha /$ MAPK (p38) signaling.

Conclusions Taken together, CTSC plays an important role in the growth and metastasis of HCC and may be a promising therapeutic target upon HCC.

\section{IDDF2019-ABS-0218 EVALUATION ON THE PROTECTION EFFECT OF THE VISMISCO IN THE LIVER DAMAGE INDUCED BY PARACETAMOL IN MICE EXPERIMENT}

Nhung Bui Thi Quynh*, Son Nguyen Van. Thainguyen university of Medicine and Pharmacy, Vietnam

\subsection{6/gutjnl-2019-IDDFabstracts.99}

Background This study was conducted to evaluate the hepatoprotective and antioxidant effects of Vismisco (extracted from Vigna radiata (L.) Wilczek, Smilax glabra roxb, Scoparia dulcis L.) in the liver damage induced by paracetamol in mice experiment.

Aim Study the hepatoprotective effect of Vismisco on the paracetamol-induced hepatotoxicity.

Methods Swiss albino mice weighing $25 \pm 2$ gram were divided into three groups of ten animals; Group 1: oral distilled water of $0.2 \mathrm{ml} / 10 \mathrm{~g}$; Group 2: oral distilled water and take paracetamol $400 \mathrm{mg} / \mathrm{kg}$; Group 3: oral Silymarin at the dose of $140 \mathrm{mg} / \mathrm{kg} /$ day and take paracetamol $400 \mathrm{mg} / \mathrm{kg}$; Group 4: oral Vismisco at the dose of $0.6 \mathrm{~g} / \mathrm{kg} / \mathrm{day}$ and take paracetamol $400 \mathrm{mg} / \mathrm{kg}$; Group 5: oral Vismisco at the dose of $1.8 \mathrm{~g} / \mathrm{kg} /$ day and take paracetamol $400 \mathrm{mg} / \mathrm{kg}$. Swiss albino mice were oral with a single dose of $400 \mathrm{mg} / \mathrm{kg}$ paracetamol to induce toxicity, while Vismisco administered in a dose of $0.6 \mathrm{~g} / \mathrm{kg} / \mathrm{day}$ and $1.8 \mathrm{~g} / \mathrm{kg} /$ day. Animals were treated daily by the oral route of administration one a day in the morning for successive 8 days and observed once daily. On the 8 th day after taking 2 hours of reagent, mice were given oral paracetamol dose of $400 \mathrm{mg} / \mathrm{kg}$. Mice were sacrificed $48 \mathrm{~h}$ after paracetamol oral to determine serum ALT, AST hepatic content of malonyl dialdehyde (MDA) and liver histopathology.

Results The 8 days pretreatment of Vismisco at the oral dose of $0,6 \mathrm{~g} / \mathrm{kg}$ and $1,8 \mathrm{~g} / \mathrm{kg}$ increases the detoxified function of the liver and reduces the increasing level of AST, ALT reduces the increasing level of hepatic MDA, reduced the inflammation, hepatocellular necrosis which was induced by paracetamol.

Conclusions Vismisco has the hepatoprotective effect from oxidative damages induced by reducing the generation of free radicals in the metabolic process of paracetamol, interrupt the lipid peroxidation of cellular membranes.

\section{IDDF2019-ABS-0221 METABOLIC-IMMUNOREGULATORY ROLE OF MTOR SIGNALING IN NAFLD- ASSOCIATED HEPATOCELLULAR CARCINOMA}

Wenshu Tang ${ }^{*}$, Jingying Zhou, Weiqin Yang, Yu Feng, Myth TS Mok, Alfred SL Cheng. The Chinese University of Hong Kong, Hong Kong

\subsection{6/gutjnl-2019-IDDFabstracts. 100}

Background and objective Hepatocellular carcinoma (HCC) is the fifth most prevalent cancer and the second leading cause of cancer deaths worldwide. Non-alcoholic fatty liver disease (NAFLD) comprises a spectrum of disorders from simple steatosis to non-alcoholic steatohepatitis (NASH). Driven by the epidemics of obesity and diabetes, it is anticipated that NAFLD will become the most important cause of HCC. Activation of mammalian target of rapamycin (mTOR) pathway in NAFLD has been shown to promote deregulated lipid/glucose metabolism, immunosuppression and tumorigenesis.

Methods To fully decipher the anti-immune surveillance functions of mTOR pathway in NAFLD-associated HCC, we performed immune cell profiling and signaling characterization in a high-fat high-carbohydrate diet (HFHC)-induced obesity and $\mathrm{NASH}$ murine model.

Results We uncovered activation of mTORC1 signaling cascade and specific reduction in cytolytic natural killer T (NKT) cells, which subsequently enhanced tumor growth of orthotopicallyimplanted HCC tumor cells. Notably, treatment of the mTORC1/C2 dual kinase inhibitor vistusertib (AZD2014), currently undergone phase I/II clinical trials, not only abrogated mTORC1/SREBP2 signaling and cholesterol levels, but also derepressed the cytolytic NKT cells in blood and liver leading to reduced tumorigenicity. Furthermore, NKT cells inactivation by a specific antibody targeting the CD1d receptor abolished anti-tumor effects of vistusertib.

Conclusions Collectively, this study elucidates the metabolicimmunosuppressive role of mTOR signaling in NAFLD-associated HCC and provides insights into the development of 
therapeutic strategies against the mTOR-reinforced NAFLDassociated HCC.

This project is supported by Collaborative Research Fund (C4045-18W) and the AstraZeneca Preclinical Oncology Research Program (2017).

\section{IDDF2019-ABS-0229 BIOCHEMICAL AND MOLECULAR EFFECTS OF $17 \beta$ ESTRADIOL ON MEMBRANE FUNCTIONS IN AGED RAT LIVER}

Pardeep Kumar*. Jawaharlal Nehru University, India

\subsection{6/gutjnl-2019-IDDFabstracts. 101}

Background Free radical production and oxidative stress are known to increase in the liver during aging and may contribute to the oxidative damage. Aging in females and males is considered as the end of natural protection against age-related diseases like osteoporosis, coronary heart disease, diabetes, Alzheimer's disease, and Parkinson's disease. These changes increase during the menopausal condition in females when the level of estradiol is decreased.

The objective of this study was to observe the changes in activities of membrane-linked ATPases $\left(\mathrm{Na}^{+} / \mathrm{K}{ }^{+}\right.$ATPase, $\mathrm{Ca}^{2}$ + ATPase), antioxidant enzymes (superoxide dismutase, glutathione-S-transferase), lipid peroxidation levels, lipofuscin content, glucose transporters 2 (GLUT 2) expression and membrane fluidity occurring in livers of female rats of 3, 12 and 24 months age groups, and to see whether these changes are restored to 3 months control levels rats after exogenous administration of $17-\beta$-estradiol (E2).

Methods The aged rats (12 and 24 months old) ( $n=8$ for each group) were given subcutaneous injection of $17 \beta$ estradiol $(0.1 \mathrm{ug} / \mathrm{g}$ body weight) daily for one month. Controls animals received an equal volume of vehicle. After 30 days of hormone treatment, experimental animals of all the groups were sacrificed and livers were isolated for further study. A detailed study was carried on membrane linked enzymes, membrane fluidity, lipofuscin and GLUT2 expression with immunohistochemistry to identify the antiaging role of E2 using biochemical, molecular and histochemical study.

Results The results obtained in the present work revealed that normal aging was associated with significant decrease in the activities of membrane-linked ATPases, antioxidant enzymes, membrane fluidity, GLUT 2 expression and an increase in lipid peroxidation and lipofuscin content in livers of aging female rats. Our data showed that exogenous administration of E2 brought these changes to near normalcy in aging female rats.

Conclusions The present study showed that E2 treatment reversed the changes to normal levels. E2 treatment may be beneficial in preventing some of the age-related changes in the liver by increasing antioxidant defenses.

\section{IDDF2019-ABS-0230 CHANGE IN THE LIPID PROFILE, LIPOGENIC AND RELATED ENZYMES IN THE LIVERS OF EXPERIMENTAL DIABETIC RATS: EFFECT OF TRIGONELLA FOENUM GRAECUM AND VANADATE}

Pardeep Kumar*, Najma Baquer. Jawaharlal Nehru University, India
Background Diabetes has been considered as one of the fastest growing epidemic worldwide; the number of people with diabetes is estimated to increase from 381.8 million in 2013 to 591.9 million in 2030. Oxidative stress in diabetic tissues is accompanied by a high level of free radicals and the simultaneously declined antioxidant enzymes status leading to cell membrane damage.

In the present study, the effect of sodium orthovanadate (SOV) and Trigonella foenum graecum seed powder (TSP) administration has been studied on hepatic glucose homeostasis, lipogenic enzymes and lipid metabolism in liver tissues of the alloxan-induced diabetic rats and to see whether the treatment with SOV and TSP is capable of reversing these effects.

Methods Diabetes was induced by administration of alloxan monohydrate $(15 \mathrm{mg} / 100 \mathrm{gm} \mathrm{b.wt}$.) and rats were treated with 2IU insulin, $0.6 \mathrm{mg} / \mathrm{ml} \mathrm{SOV}, 5 \%$ TSP in the diet and a combination of $0.2 \mathrm{mg} / \mathrm{ml} \mathrm{SOV}$ with $5 \%$ TSP separately for 21 days. Control animals were given only the vehicle. The activities of two lipogenic enzymes, glucose-6-phosphate dehydrogenase and malic enzyme; and related enzymes, hexokinase and glucose-6-phosphatase were measured in the liver cytosolic fractions of diabetic rats and diabetic rats treated separately with TSP and SOV. The total lipids, triglycerides and cholesterol levels were estimated in the livers of the diabetic and the treated rats.

Results Diabetic rats showed hyperglycemia with almost fourfold high blood glucose levels. The activities of both the lipogenic enzymes and hexokinase isozymes were significantly decreased whereas the activity of glucose-6-phosphatase was significantly increased in the diabetic liver. During diabetes, the levels of total lipids and triglycerides increased significantly with a decrease in the cholesterol levels in the liver. TSP and SOV were able to restore the altered enzyme activities to almost control levels. Rats treated with the combined dose of SOV and TSP had glucose levels comparable to controls, similar results were obtained with the triglycerides and cholesterol levels in the liver of diabetic rats.

Conclusions Our results showed that lower doses of SOV $(0.2$ $\mathrm{mg} / \mathrm{ml}$ ) could be used in combination with TSP to effectively counter diabetic alterations without any toxic side effects.

\section{IDDF2019-ABS-0234 ROLE OF MOMORDICA CHARANTIA FRUIT EXTRACT AGAINST HEPATIC FIBROSIS INDUCED BY CARBON TETRACHLORIDE IN RATS}

Sanjay Kumar*, Rajender Khanna. Vivekananda Global University, India

10.1136/gutjnl-2019-IDDFabstracts. 103

Background Momordica charantia Linn. (Karela) commonly known as Bitter melon or Bitter gourd is a tropical and subtropical climber of the family Cucurbitaceae. Its fruit is also used for the treatment of diabetes and related conditions amongst the indigenous populations of Asia, South America, India and East Africa.

The aim of the present study was to investigate the Momordica charantia fruit (MCF) extract as hepatoprotective agent verse hepatic damages caused by carbon tetrachloride $\left(\mathrm{CCl}_{4}\right)$.

Methods Male Wistar albino rats were divided into two equal groups $(n=8)$ and treated as follows: Group 1, kept as control group and orally given saline; Group 2, kept as control 